\title{
Stuve-Wiedemann syndrome with a novel mutation
}

\author{
Megan Knipe, ${ }^{1}$ Rowan Stanbury, ${ }^{1}$ Sheila Unger, ${ }^{2}$ Mallinath Chakraborty ${ }^{3}$
}

${ }^{1}$ University Hospital of Wales, Cardiff, UK

${ }^{2}$ University of Lausanne, Lausanne, Switzerland ${ }^{3}$ Department of Child Health, Cardiff University, Cardiff, UK

\section{Correspondence to} Dr Mallinath Chakraborty, chakrabortym@cardiff.ac.uk

Accepted 18 August 2015
CrossMark

\section{To cite: Knipe $M$,} Stanbury R, Unger $\mathrm{S}$, et al. BMJ Case Rep Published online: [please include Day Month Year] doi:10.1136/ bcr-2015-212032

\section{SUMMARY}

We describe a female infant born at term to consanguineous parents, with a suspicion of skeletal dysplasia in utero. At birth, she had short limbs, camptodactyly, dysphagia leading to nasogastric tube feeds, and skeletal survey demonstrating dysplasia of long bones and spine. During infancy, she also developed episodes of respiratory failure necessitating admission to intensive care, and periods of hyperhidrosis managed at home. A basic genetic screen did not reveal any abnormalities. Contact was made with the European Skeletal Dysplasia Network, and a provisional diagnosis of Stuve-Wiedemann syndrome was suggested based on this review. Specific genetic tests showed a previously unreported homozygous mutation of leukaemia inhibitory factor receptor gene, confirming the diagnosis. This is the first case with a novel mutation, reported from the UK. For paediatricians and neonatologists, the European Skeletal Dysplasia Network is a valuable resource to reach a specific diagnosis.

\section{BACKGROUND}

Skeletal dysplasias are rare general disorders of cartilage and bone; they result in disproportionate growth and short stature, ${ }^{1}$ with associated abnormalities in other organ systems. Their classification is based on a combination of molecular, biochemical and radiological criteria. ${ }^{1}$ More than 450 different skeletal dysplasias are in existence, which can be broadly classified into approximately 40 different groups based on the above diagnostic criteria. $^{2}$

Several forms of skeletal dysplasia can be diagnosed antenatally. ${ }^{3}$ However, due to the large variety of aetiology, and the rarity of individual disorders, diagnosing them in the neonatal period can prove to be challenging, except at expert centres. We present a recent case at our unit, and discuss a possible pathway for neonatologists and paediatricians to seek help when faced with an undiagnosed skeletal dysplasia.

\section{CASE PRESENTATION}

We present a case of a 41-week gestation female infant with a birth weight of $2.8 \mathrm{~kg}$ delivered vaginally. Her parents were noted to be consanguineous, with a positive family history of limb girdle type IId muscular dystrophy. The baby's mother was a 28-year-old fifth gravida, with one other live child and three previous miscarriages. Fetal anomaly scan at 21 weeks gestation in this pregnancy had shown abnormal posture of hands and feet, suggestive of arthrogryposis; an amniocentesis showed a normal female karyotype. At around 28 weeks of gestation, the tibiae of the fetus were found to be bowed, with accompanying bilateral talipes, but with good movement at that time. Towards the end of pregnancy, the mother was found to have oligohydramnios, with decreased fetal movements. Labour was induced just before 41 weeks of gestation on suspicion of prolonged rupture of membranes.

Clinical findings at birth included shortened limbs, reduced elbow extension and positional talipes. The baby was also noted to have fixed flexion deformities of her right middle and fourth fingers, with overlapping of index and little fingers, suggestive of camptodactyly (figure 1B). She was born in fair condition, but required intubation and ventilation for $2 \mathrm{~h}$ before being extubated to air.

Over the course of her neonatal admission, the baby was noted to have a difficult and possible unsafe swallow, requiring nasogastric feeding; no obvious cause was identified. Feeding was established through a nasogastric tube, and this remains in situ to date. Neurological examination demonstrated an absent suck, weak cry and weak grasp. A cranial ultrasound scan and brain MRI were both reported as normal.

A skeletal survey was performed, which showed short limb skeletal dysplasia with bowing of long bones (especially tibiae), ovoid vertebral bodies, exaggerated lumbar lordosis and an almost horizontal sacrum (figures $1 \mathrm{~A}$ and 2 ). At the time of discharge from the neonatal unit, however, the baby had no formal diagnosis.

At 6 weeks of age, she presented to the general paediatric team with shortness of breath, pallor and floppiness. She presented again at 2 months with increased work of breathing, requiring intubation, ventilation and admission to the paediatric

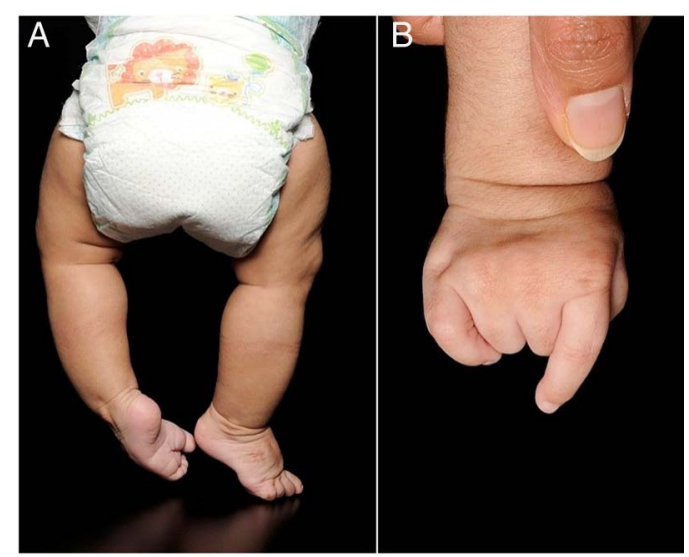

Figure 1 Clinical images. (A) Lower limbs from the back, showing bowing of legs more prominent in the left lower limb; (B) camptodactyly, shown in the right hand. 


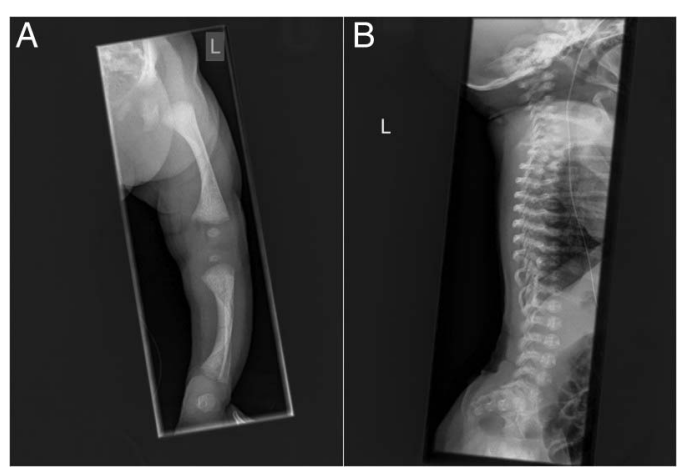

Figure 2 Skeletal survey. (A) The long bones are short with broad metaphysis, and have mild bowing which is more pronounced in the lower limbs. (B) In the spine, there are ovoid vertebral bodies, more pronounced in the lumbar spine, with a very pronounced exaggerated lumbar lordosis and almost horizontal sacrum.

intensive care unit with a metapneumovirus infection. As this was associated with lactic acidosis and hypoglycaemia, a metabolic screen was performed, the results of which were normal. The baby also underwent a bronchoscopy after one failed extubation attempt, which was reported as normal. Antireflux medications were started prior to discharge.

She was admitted again at 8 months of age to the paediatric intensive care unit for intubation and ventilation, due to respiratory distress, tachycardia and possible sepsis. Chest radiograph revealed collapse and consolidation of right upper lobe with patchy consolidation. She was managed with intravenous antibiotics, and was extubated after 5 days. To date, she has had one further admission for sepsis, not needing ventilator support. In between hospital admissions, she also developed episodes of hyperhidrosis, which were managed without admission.

In order to investigate a suspected genetic syndrome for the skeletal dysplasia, an array comparative genomic hybridisation $(\mathrm{aCGH})$ was performed, which did not reveal any abnormality; mitochondrial disorders were also excluded with specific tests.

The child had been extensively investigated by 6 months, but no specific diagnosis could be reached. Contact was made with the European Skeletal Dysplasia Network (ESDN), and she was referred to the team with a clinical summary and images after parental consent. A provisional diagnosis of Stuve-Wiedemann syndrome (SWS) was suggested by ESDN, with recommendation for specific molecular testing for mutations of the leukaemia inhibitory factor receptor (LIFR) gene. An appropriate laboratory in Germany confirmed homozygous mutation of the LIFR gene with a duplication in exon 3 at c.144_145dupGG (p. A49Gfs*6). This mutation causes a frameshift and a premature stop codon resulting either in non-sense mediated messenger RNA decay or formation of a truncated protein. This mutation was therefore considered as the cause of the patient's disease.

\section{DISCUSSION}

A specific diagnosis in suspected cases of skeletal dysplasia is important to determine prognosis, initiate surveillance for specific complications and offer counselling for family members regarding heritability. Owing to the several possible types and groups of skeletal dysplasia, which in themselves are rare diseases, ${ }^{1}$ diagnosis of specific disorders outside expert centres can be challenging. Expertise in paediatric radiology and genetics is essential in this process.
For clinicians, the ESDN can be an invaluable resource. The ESDN is a research and diagnostic network that is run by a unique group of experts. The site offers help with clinical diagnosis through an online case manager, accessible by creating a (free) account. On submission of a case history and supporting images, the panel discusses and suggests possible diagnoses and confirmatory tests. The specific diagnosis in our case was made possible only with the help of the ESDN.

SWS is an autosomal recessive bent bone skeletal dysplasia, with associated autonomic involvement. ${ }^{4}$ Most, but not all, cases of SWS have been identified to have homozygous mutations of the LIFR gene located on chromosome 5p.13.1, which is thought to be involved in fetal and neonatal bone resorption and neuronal development. Other associated features of SWS include autonomic dysfunction manifested by dysphagia, respiratory failure, pulmonary hypertension and delayed growth. Hyperthermic episodes associated with excessive and paradoxical sweating are seen; these can be life-threatening. Long-term complications include corneal opacities, along with osteopenia, pathological fractures and progressive bone deformities. Intellect is usually preserved. Prognosis is poor, with most sufferers dying in the first few months of life due to respiratory distress or hyperthermia. Management remains supportive.

Several mutations of the LIFR gene have been identified as part of SWS. However, the specific duplication in our patient, resulting in a frameshift, has not been reported previously on the Human Gene Mutation Database. ${ }^{5}$ This is possibly a private mutation carried in the family. As far as we are aware, our patient is the first reported case from the UK.

In summary, we present a case of SWS with a newly reported mutation of the LIFR gene. Diagnosis of rare skeletal dysplasias, including SWS, can be challenging, and expert groups such as the ESDN can prove invaluable in such cases. Our patient is currently 15 months old, growing steadily and making good progress in her development, although progress of gross motor function is delayed.

\section{Learning points}

Stuve-Wiedemann syndrome is a rare skeletal dysplasia, and we describe the first UK case report with a novel mutation.

- Diagnosis of specific aetiology of skeletal dysplasia can be challenging in non-expert centres.

- The European Skeletal Dysplasia Network is a freely available resource for clinicians, to help with diagnosis and confirmation.

Acknowledgements The authors would like to thank the parents of our current case, as well as all the healthcare professionals involved in her care, especially the panel members of the ESDN.

Contributors MC and SU reviewed the case and reached diagnosis and approved the final draft. All the authors supervised investigation, data collection and article draft, revised the article draft and approved the final draft.

Competing interests None declared.

Patient consent Obtained.

Provenance and peer review Not commissioned; externally peer reviewed.

\section{REFERENCES}

1 Krakow D, Rimoin DL. The skeletal dysplasias. Genet Med 2010;12:327-41.

2 Warman ML, Cormier-Daire V, Hall C, et al. Nosology and classification of genetic skeletal disorders: 2010 revision. Am J Med Genet A 2011;155A:943-68. 
3 Krakow D, Lachman RS, Rimoin DL. Guidelines for the prenatal diagnosis of fetal skeletal dysplasias. Genet Med 2009;11:127-33.

4 Mikelonis D, Jorcyk CL, Tawara K, et al. Stuve-Wiedemann syndrome: LIFR and associated cytokines in clinical course and etiology. Orphanet J Rare Dis 2014;9:34.
5 Stenson PD, Mort M, Ball EV, et al. The Human Gene Mutation Database: building a comprehensive mutation repository for clinical and molecular genetics, diagnostic testing and personalized genomic medicine. Hum Genet 2014;133:1-9.

Copyright 2015 BMJ Publishing Group. All rights reserved. For permission to reuse any of this content visit http://group.bmj.com/group/rights-licensing/permissions.

BMJ Case Report Fellows may re-use this article for personal use and teaching without any further permission.

Become a Fellow of BMJ Case Reports today and you can:

- Submit as many cases as you like

- Enjoy fast sympathetic peer review and rapid publication of accepted articles

- Access all the published articles

- Re-use any of the published material for personal use and teaching without further permission

For information on Institutional Fellowships contact consortiasales@bmjgroup.com

Visit casereports.bmj.com for more articles like this and to become a Fellow 